\title{
Mad, bad and dangerous to eat
}

\section{What the public wants remains a mystery, but food safety can't be ignored.}

\author{
When Food Kills: BSE, E. coli and \\ Disaster Science \\ by Hugh Pennington \\ Oxford University Press: 2003. 226 pp.

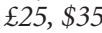 \\ Eileen Rubery
}

There is a need for a book that explains to the public what food safety is all about and that gives policy-makers and managers the conceptual framework to make sensible judgements on 'food crises' when they hit the headlines. The preface of this book does not say for whom it is written or what its aims are. But it comes from Hugh Pennington, who led Britain's inquiry into the outbreak of food poisoning caused by the bacterium Escherichia coli O157 in Wishaw in Scotland in November 1996.

Sadly, although it is good in parts, the book does not explain the basis of foodsafety assessment to the generalist. It lacks coherence and fails to develop clear lines of argument and explanation. Neither is it helpful to public-health specialists, general microbiologists or crisis managers, as it gives no advice that they could follow, nor any proposed framework for decision-making. It left me no clearer as to what Hugh Pennington thinks government or local authorities should do when faced with food scares, nor what he feels the public should think.

Instead, the book is a mêlée of good stories and fascinating anecdotes from the world of public health, loosely strung together around the stories of 'mad cow disease' (bovine spongiform encephalopathy, or BSE), new variant Creutzfeldt-Jakob disease (vCJD) and E. coli O157. The Wishaw foodpoisoning outbreak was caused by lax hygiene in a local butcher's shop: 'ready-toeat' products were contaminated with $E$. coli O157 from raw meat, and many people died. Occurring nine months after the BSE crisis, when the Conservative government had a fragile majority, the Pennington report made a number of recommendations, most of which have now been implemented.

But in When Food Kills, Pennington does not mention what to me was the really surprising outcome - that the local population sympathized with John Barr, the butcher involved, even when it became clear that he continued to distribute meat products after being identified as the source of the outbreak, thus causing many additional cases.

Why was the public so apparently illogical? Similar situations have arisen elsewhere, notably in connection with the risk of meningitis and permanent brain damage in the unborn fetus if a pregnant woman eats

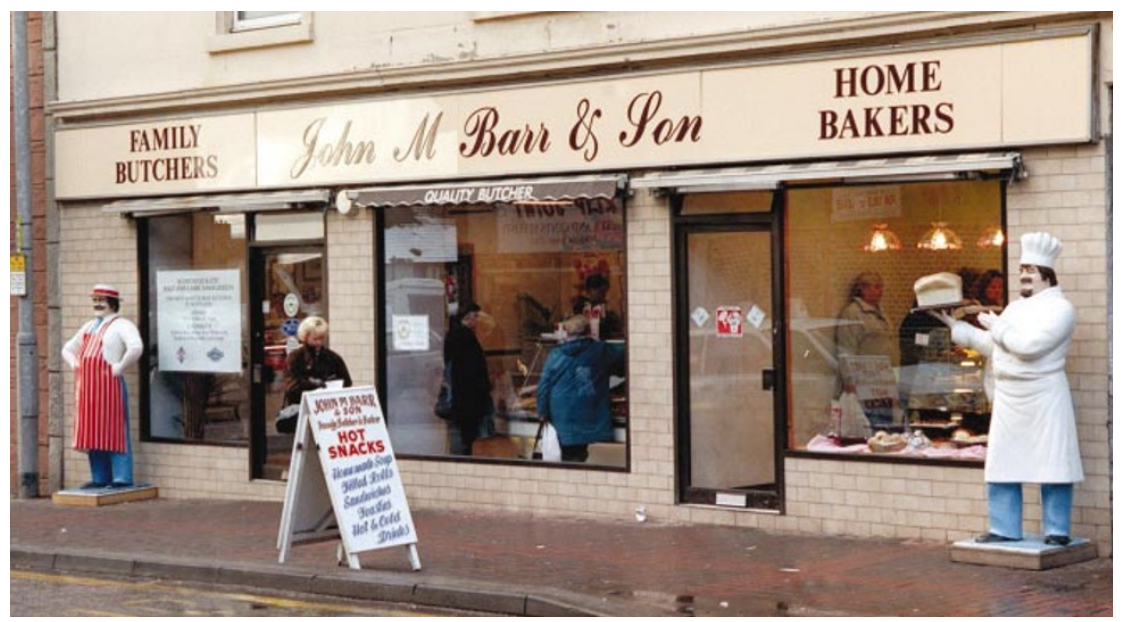

Condemned: John Barr sold lethally infected meat, but local people still supported him

cheese contaminated with Listeria and contracts listeriosis. Another example is the public's negative response to the banning of 'beef on the bone' when concerns arose about BSE prions in lymph nodes. In all these cases, it seems that the public saw heavy-handed enforcement, not an attempt to protect them. As a former policy-maker in the area of food safety, I know how difficult it is to decide what the public will perceive as the correct balance between the freedom to ply a trade and the need to protect the public.

Since the Pennington report was published, Britain's Labour government has tried to address public concerns about food safety by setting up the 'independent' Food Standards Agency (FSA). This has had some initial success (see Nature 410, 867-868; 2001), although without being tested by a really big outbreak, it is probably too early to judge its performance. Food safety is a perennial obsession of the media, which tend to focus on food crises roughly every ten years: Salmonella in eggs and botulism from hazelnut yoghurt were key concerns around 1986, then interest fell away until BSE and E. coli O157 in 1996. I predict that 2006 or 2007 will be the critical year for the FSA, and hope they are doing some forward planning.

Another problem with When Food Kills is that Pennington starts his stories from the end (the cases of illness) and works back to the beginning. So we hear about vCJD victim Stephen Churchill developing an unsteady gait, about the Foré people dying from the prion disease kuru because they eat their ancestors' brains, and about the use of ultracentrifuges to show that the infectious agent in prions is unlikely to be a nucleic acid. Only then are we told about BSE in cattle and the facts related to the BSE epidemic. This leads to a confused feeling of 'so what', rather than 'so now I understand'. A number of regulatory and inspectorial issues provide some context but few conclusions. We range as far afield as the Three Mile Island nuclear accident, train crashes from the distant and recent past, and problems in old lunatic asylums, but no strong theme or message comes from it all.

I did enjoy his quotes from what must otherwise be dry reports of public inquiries. For example, when talking of the Salmonella food-poisoning outbreak at Wakefield's Stanley Royd Hospital in 1984, Pennington reports that the kitchens had been recognized as hazardous for many years, but that the public inquiry concluded that planning activities were a "remarkable example of what well-intentioned individuals can fail to achieve unless someone is charged with the responsibility of ensuring that careful attention to detail does not lead to a complete cessation of all activity other than the production of paper". Unfortunately, today's well-intentioned emphasis on consultation and team-working probably exacerbates, rather than ameliorates, this problem.

Then Pennington quotes from the report of the inquiry into the 1966 Aberfan landslide disaster: "We found that many witnesses ... had been oblivious" to the dangerous state of the tips. "It did not enter their consciousness. They were like moles being asked about the habits of birds."

Individually these stories are entertaining and provide memorable examples of the human propensity to ignore danger signals until it is too late. Indeed, one ends up surprised that disaster did not strike far earlier, or more often, given the sagas of negligence and misunderstandings detected once an 
investigation begins. But the overall impact is reduced by the lack of critical analysis of the cases, and unfocused and superficial discussion of the difficulties of keeping alert those charged with protecting us from rare but serious dangers.

Although overall the book disappoints, I know I will be using Pennington's quotes in my lectures in future. Indeed, one quote from the preface will remain with me. In Shakespeare's Twelfth Night, the foolish knight Sir Andrew Aguecheek anticipates the BSE crisis by saying "but I am a great eater of beef and I believe that does harm to my wits," to which Sir Toby, his companion, replies: "No question." The bard, as ever, was far ahead of his time.

Eileen Rubery is at the Judge Institute of

Management, University of Cambridge,

Trumpington Street, Cambridge CB2 1AG, UK.

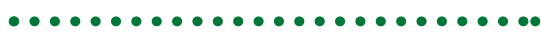

Prime time for mathematics

Prime Obsession: Bernhard

Riemann and the Greatest

Unsolved Problem in Mathematics by John Derbyshire

Joseph Henry: 2003. 456 pp. \$24.95, £19.95

The Music of the Primes: Why an Unsolved Problem in Mathematics Matters

by Marcus du Sautoy

Fourth Estate: 2003. 335 pp. 118.99

HarperCollins: 2003. \$24.95

\section{W. T. Gowers}

For many years, the Riemann hypothesis has been regarded as one of the most important unsolved problems in mathematics. If it could be proved, it would go a long way towards demonstrating what observation already suggests - that in many ways prime numbers are distributed as if they had been chosen randomly. Popular science is big business these days, and there is a rapidly developing sub-genre of books about famous mathematical problems, either unsolved or recently solved. So it was inevitable that attention would turn to the Riemann hypothesis, especially as it now carries a bounty of a million dollars, offered by the Clay Mathematics Institute. And, sure enough, now there are three popular books on the subject: Dr. Riemann's Zeros by Karl Sabbagh (Atlantic: 2002) and the two reviewed here.

Unfortunately, and in contrast to other mathematical problems such as Fermat's last theorem, Kepler's conjecture and the travelling-salesman problem, the basic statement of the Riemann hypothesis - that the nontrivial zeros of Riemann's zeta function all have real parts equal to one half — is rather sophisticated and difficult to explain to a general audience. The zeta function is defined by the infinite series

$$
\zeta(s)=1^{-s}+2^{-s}+3^{-s}+4^{-s}+\ldots
$$

so it is important to understand when a series such as this makes sense. Next, we need to understand Riemann's crucial innovation, which was to extend the definition of the zeta function to include complex numbers (the function had already been considered by Euler for real $s$ ): even readers who are familiar with $i$, the square root of -1 , may be baffled by the idea of $2^{-i}$. After coming to terms with all that, one learns that the expression for the zeta function does not make sense when the real part of $s$ lies between 0 and 1 . Nevertheless, there is a way of making sense of the zeta function itself in this region, and, remarkably, its behaviour has important consequences for the distribution of prime numbers. Since it is the seeming randomness of the primes that lies behind much of modern cryptography, and in particular behind the security of the Internet, understanding these mysterious numbers is of the utmost importance.

In his book Prime Obsession, John Derbyshire sets himself the heroic task of explaining the Riemann hypothesis to readers who have no mathematical background beyond perhaps a basic fluency at rearranging bits of algebra and a half-forgotten exposure

to calculus. Although all the steps are there, he is attempting the impossible: to learn a subject as hierarchical as mathematics, you have to understand and digest one level thoroughly before you can move on to the next. Indeed, he almost admits this in his introduction: "If you don't understand the Hypothesis after finishing my book, you can be pretty sure you will never understand it."

But it would be quite wrong to judge the book a failure for this reason. In common with almost all books of this kind, there are parts that you will skim, and which these are will depend on your mathematical background — but the bits you do read will be extremely well explained. A major and most unusual strength of the book, which even experts will enjoy, is a sort of intimacy between the author and the zeta function itself. Not content with the abstract definition, he tabulates values and draws numerous diagrams (not easy, as the four dimensions of the graph must be represented on a single page) to convey to the reader the intuitive feel for the function that he himself quite clearly has.

Marcus du Sautoy's entertaining book The Music of the Primes is aimed at the more popular end of the market and looks certain to be a great success. He is less ambitious than Derbyshire in what he tries to explain, and is wider in his focus, covering prime numbers in general and not just the Riemann hypothesis. Although many of his anecdotes, such as what Ramanujan said about the number 1729 , will be familiar to readers of other popular mathematics books, there are many other good ones that are less well known. I particularly recommend the enlightening story of how Don Zagier, a contemporary number theorist, changed his mind and started to believe that the Riemann hypothesis was true - and what that had to do with the most expensive bottle of wine ever.

The language used by du Sautoy is more hyperbolic than Derbyshire's - for example, the word 'stunning' makes regular appearances. He also likes to use certain metaphors repeatedly. Most notably, instead of saying, "the zeros of Riemann's zeta function all lie on the critical line", he prefers, "the points at sea level in Riemann's imaginary landscape all lie on the magic ley line". When such metaphors have been used often enough, they can end up simply as substitutes for more standard terms (and, indeed, many standard mathematical words were themselves chosen for their metaphorical suggestiveness). Derbyshire has the same habit — for him, Euler's product formula is the "golden key", the turning of which is presented as one of the high points of Riemann's argument.

The chapters of Derbyshire's book alternate between mathematical ones and more historical ones, a device that works well. Riemann is one of the few undoubted geniuses of the subject, and had an extraordinary and all-pervasive influence on mathematics. As well as the Riemann zeta function, he came up with the Riemann sphere, Riemann surfaces, Riemannian manifolds, the Cauchy-Riemann equations, the Riemann integral and numerous theorems that also bear his name. He was a shy man from a relatively humble background and much of his short life was a struggle against poverty, illness and depression. It is hard not to be moved that such a life left the world so enriched.

W. T. Gowers is at the Centre for Mathematical Sciences, University of Cambridge, Wilberforce Road, Cambridge CB3 OWA, UK. 